\title{
D0 Measurement of the Dijet Azimuthal Decorrelations
}

\author{
Marek Zieliński ${ }^{1}$ \\ University of Rochester, Rochester, NY, U.S.A.
}

\begin{abstract}
.
We present the D0 measurement of correlations in the azimuthal angle between the two largest transverse momentum jets produced in $p \bar{p}$ collisions at a center-of-mass energy $\sqrt{s}=1.96 \mathrm{TeV}$ in the central rapidity region [1]. The results are based on an inclusive dijet event sample corresponding to an integrated luminosity of $150 \mathrm{pb}^{-1}$. Data is in good agreement with next-to-leading order (NLO) pQCD calculations, and with tuned PYTHIA, HERWIG, ALPGEN and SHERPA event generators.
\end{abstract}

The proper description of multi-parton radiation is crucial for a wide range of precision measurements as well as for searches for new physical phenomena. The azimuthal difference $\Delta \phi$ between two leading jets in an event is a clean and simple probe of radiation effects. In the absence of radiation $\Delta \phi=\pi$; soft radiation causes small deviations from $\pi$, while $\Delta \phi$ significantly lower than $\pi$ indicates the presence of hard radiation, such as a third jet with high $p_{T}$. The range $\Delta \phi<2 \pi / 3$ is populated by events with 4 or more hard jets Distributions in $\Delta \phi$ allow testing the QCD descriptions across a range of jet multiplicities without requiring the reconstruction of additional jets and offer a way to examine the transition between soft and hard QCD processes based on a single observable. The observable is defined as the differential dijet cross section in $\Delta \phi$, normalized by the dijet cross section integrated over $\Delta \phi$ in the same phase space $\left(1 / \sigma_{\text {dijet }}\right)\left(d \sigma_{\text {dijet }} / d \Delta \phi\right)$. Theoretical and experimental uncertainties are reduced in this construction. Jets are defined using an iterative seed-based cone algorithm (including mid-points) with radius $R_{\text {cone }}=0.7$ [2] at parton, particle, and experimental levels.

Four analysis regions were defined based on the jet with largest $p_{T}$ in an event $\left(p_{T}^{\max }\right)$ with the requirement that the trigger efficiency be at least $99 \%$. The second leading $p_{T}$ jet in each event was required to have $p_{T}>40 \mathrm{GeV}$ and both jets were required to have central rapidities with $|y|<0.5$. Several event selection criteria reduced effects of misreconstruction and the backgrounds from cosmic rays, as well as from electrons, photons and noise mimicking jets [1]. The overall selection efficiency was typically $\approx 80 \%$. The jet energy calibration was the largest contribution to systematic uncertainty.

The corrected data are presented in Fig. 1 as a function of $\Delta \phi$ in four ranges of $p_{T}^{\max }$. The spectra are strongly peaked at $\Delta \phi \approx \pi$; the peaks are narrower at larger values of $p_{T}^{\max }$. Overlaid on the data points in Fig. 1 (left) are the results of pQCD calculations

\footnotetext{
${ }^{1}$ For the D0 Collaboration.
} 

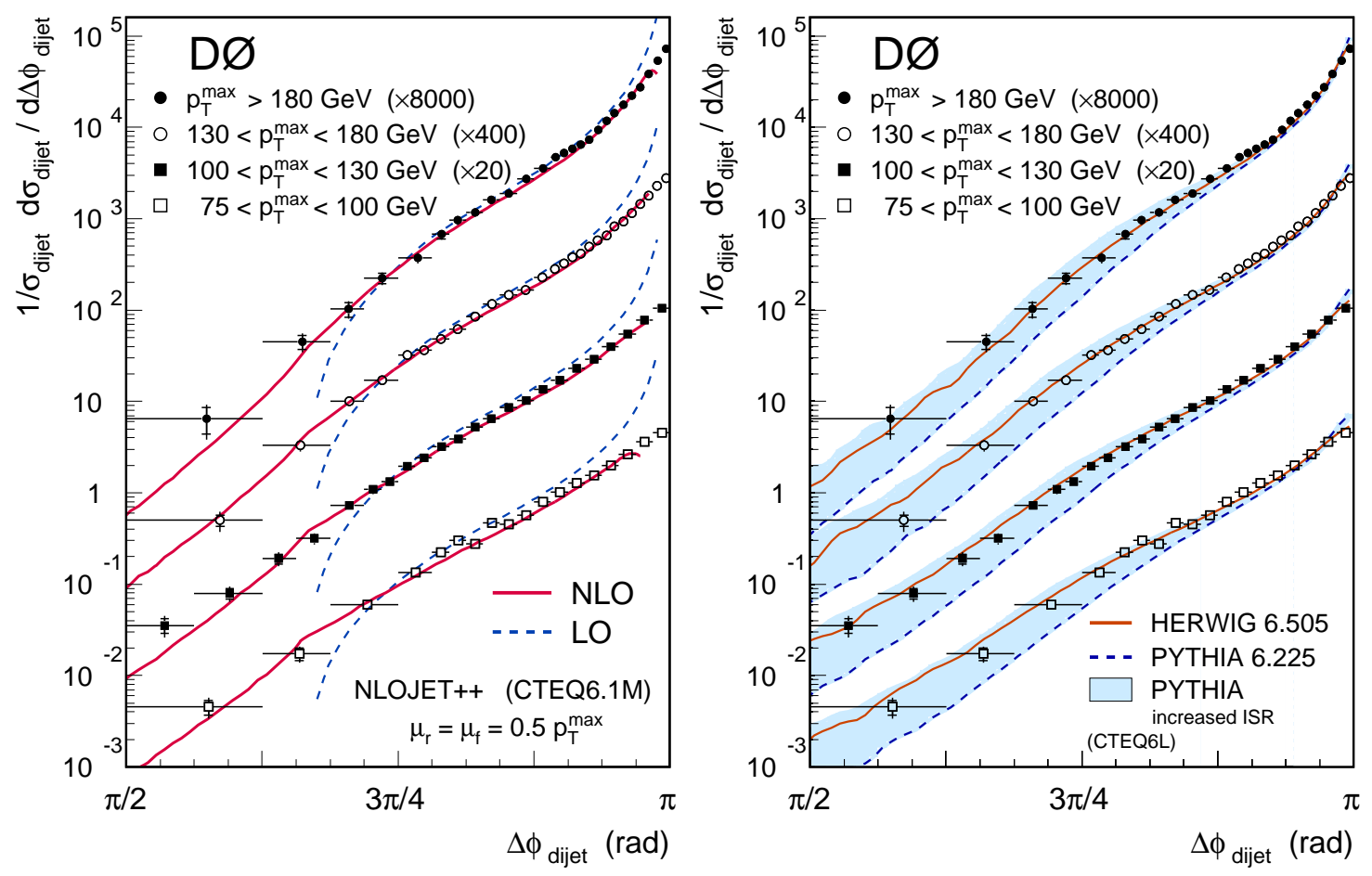

FIGURE 1. Left: The $\Delta \phi$ distributions in different $p_{T}^{\max }$ ranges. Data and predictions with $p_{T}^{\max }>100$ $\mathrm{GeV}$ are scaled by successive factors of 20 for purposes of presentation. The solid (dashed) lines show the NLO (LO) pQCD predictions. Right: Analogous presentation of results from HERWIG and PYTHIA overlaid on the data.

obtained using the parton-level event generator NLOJET++ [3]; NLO pQCD provides a good description of data, while the leading-order (LO) calculation has a limited applicability. The pQCD calculations are insensitive to hadronization corrections and the underlying event effects [4]].

Monte Carlo event generators, such as HERWIG [5] and PYTHIA [6], use $2 \rightarrow 2$ LO pQCD matrix elements with phenomenological parton-shower models to simulate higher order QCD effects. Results from HERWIG (version 6.505) and PYTHIA (version 6.225), both using default parameters, are compared to the data in Fig.11(right). HERWIG describes the data well over the entire $\Delta \phi$ range including $\Delta \phi \approx \pi$. PYTHIA with default parameters describes the data poorly - the distribution is too narrowly peaked at $\Delta \phi \approx \pi$ and lies significantly below the data over most of the $\Delta \phi$ range. The shaded bands in Fig. 1. (right) indicate the range of variation when the maximum allowed virtuality in the initial-state shower is smoothly increased from the current default by a factor of four. These variations clearly demonstrate the sensitivity of this measurement. Global efforts to tune Monte Carlo event generators can benefit from including our data to constrain the related model parameters.

Recent developments in event generators aim at improving the description of processes involving multiple jets. These approaches combine exact LO PQCD matrix elements for multi-parton production with parton-shower models; care must be taken to avoid double counting of equivalent phase-space configurations. The $\Delta \phi$ distributions 

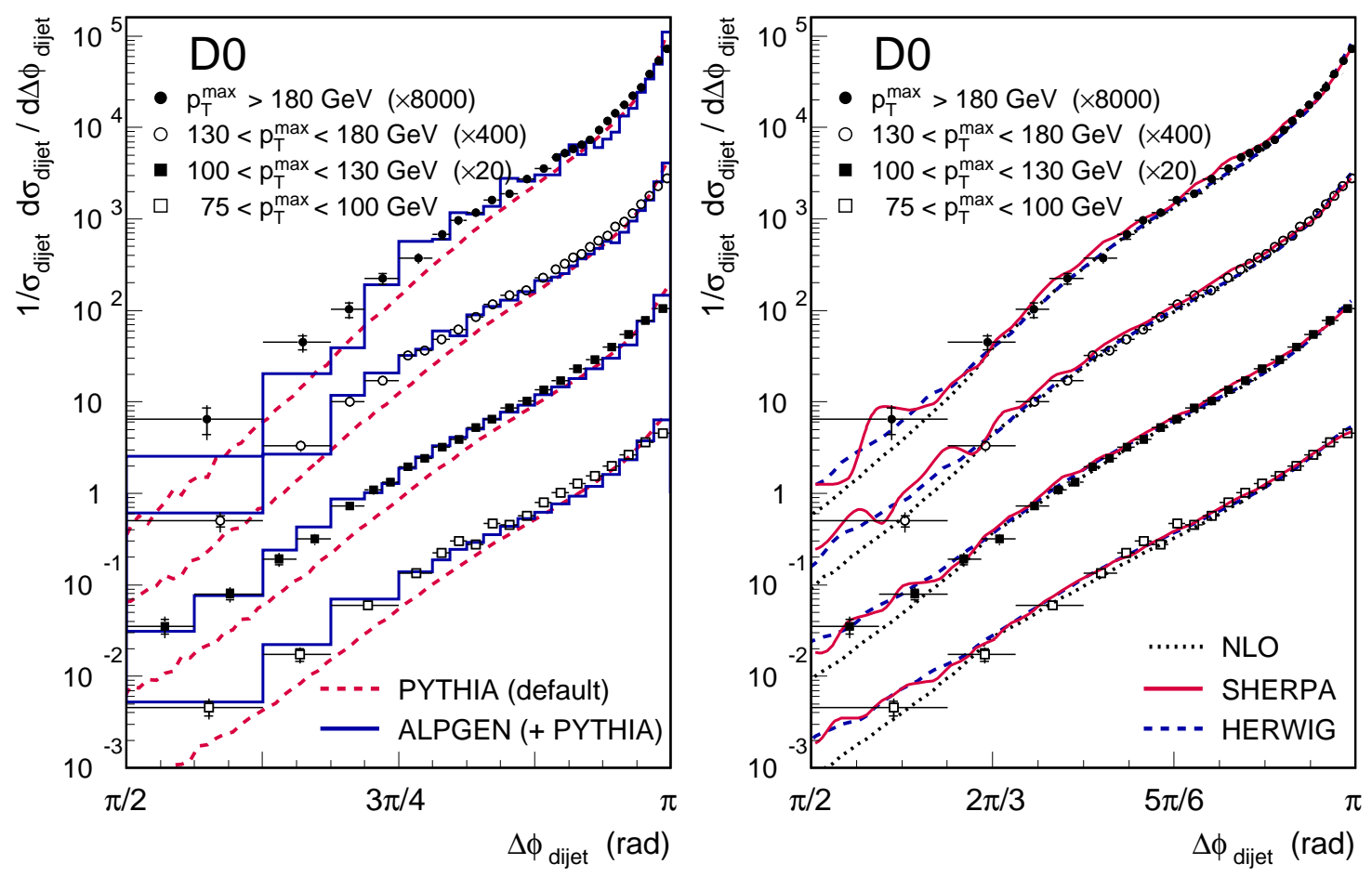

FIGURE 2. Left: Comparison of predictions from ALPGEN (interfaced to default PYTHIA) with data. Right: Comparison of predictions from SHERPA to data, and to predictions from NLOJET++ and from HERWIG.

are sensitive to a range of jet multiplicities and provide a test of such techniques. Two such generators, ALPGEN [7] and SHERPA [8], are shown to be in good agreement with data (Fig. 2), thus enhancing confidence in their applications to other processes.

\section{ACKNOWLEDGMENTS}

We thank W. Giele, T. Gleisberg, F. Krauss, M. Mangano, Z. Nagy, M. H. Seymour, and T. Sjöstrand for discussions.

\section{REFERENCES}

1. V. M. Abazov et al. [D0 Collaboration], Phys. Rev. Lett. 94, 221801 (2005).

2. G. C. Blazey et al., hep-ex/0005012

3. Z. Nagy, Phys. Rev. Lett. 88, 122003 (2002); Phys. Rev. D 68, 094002 (2003).

4. M. Wobisch [D0 Collaboration], AIP Conf. Proc. 753, 92 (2005) [hep-ex/0411025].

5. G. Marchesini et al., Comp. Phys. Comm. 67, 465 (1992); G. Corcella et al., JHEP 0101, 010 (2001).

6. T. Sjöstrand et al., Comp. Phys. Comm. 135, 238 (2001).

7. M. L. Mangano et al., JHEP 0307, 001 (2003).

8. T. Gleisberg et al., Phys. Rev. D 72, 034028 (2005) and references therein. 